\title{
Relationship between the movement characteristics of human spermatozoa and their ability to penetrate cervical mucus and zona-free hamster oocytes
}

\author{
R. J. Aitken, M. Sutton, P. Warner and D. W. Richardson \\ MRC Reproductive Biology Unit, Centre for Reproductive Biology, 37 Chalmers Street, \\ Edinburgh EH3 9EW, U.K.
}

\begin{abstract}
Summary. In a group of normospermic donors exhibiting hamster oocyte penetration scores of $0-100 \%$, multiple regression analysis indicated that only $20 \%$ of the variation in fertilizing potential could be explained by differences in the movement characteristics of the spermatozoa following incubation in vitro. When the movement characteristics of the spermatozoa in semen were considered this figure was reduced to $6.8 \%$ as a result of significant differences in the motility patterns exhibited by the seminal and post-incubation sperm populations.

A much closer relationship was observed between the movement characteristics of human spermatozoa in semen and their ability to penetrate cervical mucus. When differences in motile sperm densities were taken into account, $76 \%$ of the variation in cervical mucus penetration could be accounted for by the existence of linear correlations with certain aspects of sperm movement (multiple $R=0.874$ ). Of the various attributes of sperm motility measured (linear velocity of progression, frequency of rotation, amplitude of sperm head displacement, $\%$ rolling and $\%$ yawing), a failure to exhibit an adequate amplitude of lateral sperm head displacement was consistently found to be the most significant factor determining the success of sperm-cervical mucus interaction $\left(R^{2}=0.53\right)$.
\end{abstract}

\section{Introduction}

The development of in-vitro techniques to assess the functional competence of human spermatozoa is of direct relevance to the clinical diagnosis of male infertility and the evaluation of new approaches to male contraception. Sperm motility is an attribute of semen quality which is thought to be of critical importance in the definition of fertility, in relation to the achievement of fertilization (Yanagimachi, 1970; Aitken, Best, Richardson, Djahanbakhch \& Lees, 1982a; Aitken et al., 1982b, c, 1983b; Bedford, 1983) and ascent of the female reproductive tract (Mortimer \& Templeton, 1982), particularly at the level of the cervix (David et al., 1979; Schats, Aitken, Templeton \& Djahanbakhch, 1984). Several techniques have been developed to measure objectively the detailed movement characteristics of human spermatozoa (Atherton, Radany $\&$ Polakoski, 1977) of which cinematography (David, Serres \& Jouannet, 1981), videomicrography (Katz \& Overstreet, 1981) and time-exposure photomicrography (Overstreet, Katz, Hanson \& Fonseca, 1979) appear to give the most unambiguous results. Objective assessment of the movement characteristics of human spermatozoa is not an end in itself, however; such measurements only take on meaning when they can be related to sperm function (Serres, Feneux, Jouannet \& David, 1984). The purpose of this study was to assess the relationships between sperm motility and functional competence, by using in-vitro tests which have been shown to produce results relating to fertility in vivo (Ulstein, 1973; Aitken, Best, Warner \& Templeton, 1984) and which purport to measure the ability of human spermatozoa to fertilize (Yanagimachi, Yanagimachi \& Rogers, 1976) and to penetrate cervical mucus (Katz, Overstreet \& Hanson, 1980) respectively. 


\section{Materials and Methods}

Semen donation. The semen samples were produced by masturbation into sterile plastic containers and were subjected to a detailed analysis in the laboratory within $1.5 \mathrm{~h}$. All donors were advised on the need to abstain from intercourse for $48 \mathrm{~h}$ before producing a sample and to maintain the sample at body heat during transit to the laboratory. All samples were initially analysed according to the criteria laid down by the World Health Organisation (1980) to obtain a conventional semen profile describing the volume and physical appearance of the sample as well as the number, morphology and motility of the spermatozoa. All of the 52 donors used in this study were 'normal' healthy volunteers exhibiting 'normal' semen profiles (sperm concentration $>20 \times 10^{6} / \mathrm{ml}$; progressive motility $>40 \%$; normal morphology $>40 \%$ ). They constituted a suitable population on which to realize the objectives of this study by virtue of the wide range of competence they exhibited in the functional assays described below.

Sperm movement characteristics. The movement characteristics of the spermatozoa were determined by time-exposure photomicrography (Overstreet et al., 1979; Aitken et al., 1982a, b, c). The spermatozoa were photographed at $37^{\circ} \mathrm{C}$, under dark-field illumination using an exposure time of $1 \mathrm{sec}$. The sperm tracks were measured to determine the linear velocity of progression, frequency of rotation and amplitude of lateral sperm head displacement. In addition the patterns of sperm movement were classified as 'rolling', 'yawing' or 'minimal displacement' (in which the successive rotations of the sperm head followed the same linear path giving a displacement of $<4.5 \mu \mathrm{m}$ ). The results were expressed as a proportion of the populations of total motile or progressively motile $(>25 \mu \mathrm{m} / \mathrm{sec})$ spermatozoa and all measurements were made on 20 randomly selected tracks omitting any cells leaving the microscopic field.

Penetrating ability. The fertilizing potential of the spermatozoa was assessed at a concentration of $10 \times 10^{6}$ cells $/ \mathrm{ml}$ using the zona-free hamster oocyte penetration test originally described by Yanagimachi et al. (1976) and modified by Aitken, Wang, Lui, Best \& Richardson (1983a). This procedure incorporated a 3-h capacitation period in Medium BWW (Biggers, Whitten \& Whittingham, 1971), the osmolarity of which had been raised to $410 \mathrm{mosmol} / \mathrm{kg}$ by the addition of $\mathrm{NaCl}$. At the end of the capacitation phase the spermatozoa were pelleted by centrifugation at $500 \mathrm{~g}$ for $5 \mathrm{~min}$ and resuspended in normosmotic Medium BWW (305 mosmol $/ \mathrm{kg}$ ) before the addition of zona-free hamster oocytes. The spermatozoa were incubated with the oocytes for a further $3 \mathrm{~h}$, after which the latter were mounted on glass slides, compressed to a depth of about $30 \mu \mathrm{m}$ and examined by phase-contrast microscopy for the presence of decondensing sperm heads within the vitellus. The percentage of oocytes penetrated was taken as the penetration rate and at least 20 oocytes were used to assess each sample. The penetration results obtained with this protocol exhibit a correlation coefficient of 0.949 with those obtained after a 7 -h preincubation period in normosmotic medium (Aitken et al., 1983a).

Cervical mucus penetration. Quantitative assessment of the success of sperm interaction with cervical mucus was obtained using a preparation of oestrous bovine cervical mucus stored at $-20^{\circ} \mathrm{C}$ for 6-8 weeks and the in-vitro procedures described by Katz et al. (1980). The cervical mucus was loaded into flat capillary tubes with a depth of $300 \mu \mathrm{m}$ (Camlab, Cambridge) using the negative pressure generated by a $10 \mathrm{ml}$ syringe (Katz et al., 1980). Once one end of these tubes had been sealed with Critoseal (Lancet, Sherwood Medical, U.S.A.) the tubes were broken at a point 27 $\mathrm{mm}$ from the Critoseal/cervical mucus junction, to give an internal volume of $25 \mu \mathrm{l}$. Duplicate tubes prepared in this manner were equilibrated for $20 \mathrm{~min}$ at $37^{\circ} \mathrm{C}$ in an humidified chamber containing an atmosphere of $5 \% \mathrm{CO}_{2}-95 \%$ air. The open end of each tube was then gently lowered into a $100 \mu \mathrm{l}$ sample of semen, inclined at an angle of $45^{\circ}$ and incubated for $30 \mathrm{~min}$ in an atmosphere of $5 \% \mathrm{CO}_{2}-$ $95 \%$ air.

After incubation the capillary tubes were removed from the semen samples and carefully rinsed 
in a mucolytic solution containing $0.1 \%$ 2-mercaptoethanol and $10 \%$ formalin in Dulbecco's phosphate-buffered saline (Flow Laboratories, Irvine, U.K.) to remove residual spermatozoa from the external surface of the tubes and non-penetrating spermatozoa from the cervical mucus interface. The mucus was then extruded into $475 \mu$ l of the mucolytic solution and the tubes were repeatedly washed to ensure optimal recovery of the spermatozoa. The concentration of spermatozoa in the solubilized mucus was estimated using an improved Neubauer haemocytometer after which the success of sperm interaction with the cervical mucus was calculated according to the formula (Katz et al., 1980):

$$
\mathrm{PSC}=400 \frac{\mathrm{Cm}}{\mathrm{Cs}} \cdot \frac{1 / \mathrm{t}}{\mathrm{Vs}}
$$

where PSC $=$ percentage successful collisions $; 400=$ a statistical factor $(4) \times 100 ; \mathrm{Cm}=$ concentration of motile spermatozoa in mucus $\left(10^{6} / \mathrm{ml}\right) ; \mathrm{Cs}=$ concentration of motile spermatozoa in semen $\left(10^{6} / \mathrm{ml}\right) ; 1=$ length of capillary tube $(\mu \mathrm{m}) ; \mathrm{t}=$ time $(\mathrm{sec}) ; \mathrm{Vs}=$ mean linear velocity of progression $(\mu \mathrm{m} / \mathrm{sec})$ as determined by time exposure photomicrography.

Statistical Analysis. The data were analysed using the Statistical Package for the Social Science's multiple regression programme (Nie, Hull, Jenkins, Steinbrenner \& Bent, 1975). The assumptions underlying multiple regression analysis (linearity of the regression, normal distribution and homogeneity of variance) were investigated by scattergram plots of the raw data and examination of the distribution of residuals. In the text the following conventions have been used; $r=$ simple correlation coefficient, $R=$ multiple correlation coefficient and $R^{2}=$ the proportion of variance of $y$ explained by the multiple regression equation.

\section{Results}

\section{Hamster oocyte penetration}

An analysis was made of the relationship between hamster oocyte penetration and the movement characteristics in semen, and after capacitation in vitro, of the spermatozoa of 35 donors. This group of donors gave a mean \pm s.e.m. hamster oocyte penetration rate of $56.5 \pm 5.3 \%$ with a range of $0-100 \%$. Calculation of correlation coefficients describing the relationship between the movement characteristics of the spermatozoa in semen and their subsequent ability to penetrate zona-free hamster oocytes did not reveal the presence of any significant regressions. Multiple regression analysis using the movement characteristics of the total motile seminal population as the independent variables produced an $R$ value of 0.22 after 5 variables had been incorporated into the regression equation. When the movement characteristics of the progressively motile spermatozoa in semen were employed as the independent variables, a similar $R$ value of 0.26 was obtained following the incorporation of 5 variables. These results indicate that, at best, only $6.8 \%$ of the variation in hamster oocyte penetration scores could be explained by the existence of linear correlations with the movement characteristics of the spermatozoa in semen.

Once the spermatozoa had been washed free of seminal plasma and incubated in vitro to effect capacitation, the movement characteristics of the spermatozoa changed significantly in relation to their movement in semen (Table 1). The post-incubation sperm populations contained significantly fewer motile cells $(P<0.001)$ exhibiting higher velocities $(P<0.001)$ with an increased frequency of rotation $(P<0.001)$. The patterns of sperm motility also changed significantly, resulting in an increased proportion of spermatozoa exhibiting a rolling swimming motion $(P<0.01)$ and a decreased incidence of yawing $(P<0.01)$. Although there was a tendency for the post-incubation sperm populations to exhibit a larger amplitude of lateral sperm head displacement than their counterparts in semen, this trend was not significant (Table 1).

These post-incubation patterns of sperm motility exhibited a slightly improved correlation with 
Table 1. Comparison of the movement characteristics of the total motile sperm population in human semen and after incubation in vitro $(n=35)$

\begin{tabular}{lrc}
\hline \multicolumn{1}{c}{ Movement characteristics } & Semen & After incubation \\
\hline Motility $(\%)$ & $65 \cdot 8 \pm 1 \cdot 4$ & $50 \cdot 7 \pm 2 \cdot 1^{* * *}$ \\
Mean velocity $(\mu \mathrm{m} / \mathrm{sec})$ & $32 \cdot 4 \pm 0 \cdot 7$ & $43 \cdot 8 \pm 1 \cdot 4^{* * *}$ \\
Amplitude of lateral displacement $(\mu \mathrm{m})$ & $6 \cdot 3 \pm 0 \cdot 2$ & $6 \cdot 4 \pm 0 \cdot 2$ \\
Frequency of rotation $(/ \mathrm{sec})$ & $5 \cdot 3 \pm 0 \cdot 2$ & $8 \cdot 7 \pm 0 \cdot 4^{* * *}$ \\
Rolling $(\%)$ & $47 \cdot 4 \pm 3 \cdot 0$ & $60 \cdot 7 \pm 3 \cdot 3^{* *}$ \\
Yawing $(\%)$ & $6 \cdot 4 \pm 1 \cdot 1$ & $1 \cdot 8 \pm 0 \cdot 6^{* *}$ \\
Minimal displacement $(\%)$ & $46 \cdot 1 \pm 3 \cdot 4$ & $37 \cdot 4 \pm 3 \cdot 5$ \\
\hline
\end{tabular}

Values are means \pm s.e.m.

** $P<0 \cdot 01, * * * P<0 \cdot 001$.

the fertilizing ability of the spermatozoa recorded in the zona-free hamster oocyte assay. Hence simple correlations were observed for the percentage of motile spermatozoa in the incubation media $(r=0.370 ; P<0.05)$ and the concentration of spermatozoa exhibiting an amplitude of lateral sperm head displacement of $<10 \mu \mathrm{m}(r=0.330 ; P<0.05)$. Multiple regression analysis gave an $R$ value of 0.437 after 5 variables had been incorporated into the regression equation including $\%$ motility, frequency of rotation, $\%$ yawing, mean amplitude of lateral displacement and $\%$ rolling spermatozoa with an amplitude of lateral displacement of $<10 \mu \mathrm{m}$. Hence on the basis of these variables about $20 \%$ of the variation in hamster oocyte penetration scores could be explained by differences in post-incubation movement characteristics. None of the 5 samples exhibiting subnormal hamster oocyte penetration scores of $<10 \%$ (Rogers et al., 1979; Aitken et al., 1982a) in the study population were correctly identified by this multiple regression analysis. For predicted penetration results of $48 \cdot 7,30 \cdot 9,48 \cdot 2,43 \cdot 4$ and $43 \cdot 3 \%$, the observed penetration values were $0,0,5 \cdot 5$, 8.3 and $10.0 \%$ respectively.

\section{Cervical mucus penetration}

The relationship between the movement characteristics of human spermatozoa in semen and their ability to penetrate cervical mucus was assessed for 17 donors exhibiting a mean \pm s.e.m. PSC score of $47 \cdot 1 \pm 6.0 \%$ with a range of $14.0-102.0 \%$. When correlation coefficients were calculated describing the relationship between the movement characteristics of the spermatozoa and the PSC scores, the degree of lateral displacement of the sperm head was found to be the single most important criterion determining the success of sperm-cervical mucus interaction. This relationship was expressed as a positive correlation with the mean width of lateral displacement (total motile population, $r=0.645, P<0.01$; progressively motile population, $r=0.626$; $P<0.01)$ and as a negative correlation with the proportion of spermatozoa exhibiting either minimal lateral displacement of the sperm head (total motile population, $r=-0.646, P<0.01$; progressively motile population, $r=-0.592, P<0.01$ ) or a displacement of $<10 \mu \mathrm{m}$ (total motile population, $r=-0.701 ; P<0.001)$. The frequency with which the 'yawing' pattern of sperm progression was encountered did not correlate with the PSC values although significant positive correlations were observed with the 'rolling' pattern which, by definition, involves the expression of significant lateral displacement of the sperm head (total motile population, $r=0.508 ; P<0.05$; progressively motile population $r=0.493 ; P<0.05$ ).

In the light of these results indicating that certain attributes of sperm movement are of importance in determining the success of sperm-cervical mucus interaction, a multiple regression analysis was performed using the PSC values as the dependent variable. In this analysis a multiple correlation coefficient of 0.956 was observed between the PSC scores and sperm movement, on the basis of 10 variables describing various aspects of sperm motility. The most informative variables in this analyses were the proportion of motile spermatozoa failing to exhibit significant lateral 
displacement $\left(R^{2}=0.313\right)$ and the proportion of progressively motile spermatozoa exhibiting an amplitude of displacement of $<10 \mu \mathrm{m}\left(R^{2}\right.$ change $\left.=0.182\right)$, both of which were negatively correlated with cervical mucus penetration. When the number of independent variables was limited by considering only the total motile population, and the number of variables permitted in the multiple regression equation was limited to 5 , a multiple $R$ of 0.662 was obtained on the basis of the variables listed in Table 2 . In this analysis the proportion of motile spermatozoa failing to exhibit significant lateral displacement was again the most informative variable $\left(R^{2}=0 \cdot 313\right)$. When the regression was confined to the movement characteristics of the progressively motile sperm population, the multiple $R$ value obtained after 5 variables had been incorporated in the equation was virtually unchanged at 0.635 . Scattergrams plotting the independent variables against the PSC values indicated that, for the variables incorporated into the regression equations, the relationships were approximately linear and would not be improved by transformation.

Table 2. Multiple regression of percentage of successful collision (PSC) scores on the movement characteristics of the total motile sperm population in human semen

\begin{tabular}{lccr}
\hline \multicolumn{1}{c}{ Variable } & Multiple $R$ & $R^{2}$ change & $\begin{array}{c}\text { B } \\
\text { (non-standardized) }\end{array}$ \\
\hline Minimal displacement $(\%)$ & 0.559 & 0.313 & -0.857 \\
Frequency of rotation $(/ \mathrm{sec})$ & 0.612 & 0.061 & $-2 \cdot 429$ \\
Motility $(\%)$ & 0.641 & 0.037 & 0.514 \\
Mean velocity $(\mu \mathrm{m} / \mathrm{sec})$ & 0.656 & 0.018 & $-1 \cdot 791$ \\
Mean amplitude of displacement $(\mu \mathrm{m})$ & 0.662 & 0.008 & 4.492 \\
Constant & & & 113.526 \\
\hline
\end{tabular}

The use of PSC values as the dependent variable is complicated by the fact that 2 of the independent variabies (motility and total mean velocity) are used in its calculation. To overcome this problem multiple regressions were performed in which the concentration of spermatozoa in the mucus $(\mathrm{Cm})$, rather than the PSC score, was used as the dependent variable. Analysis of scattergrams plotting the relationship between $\mathrm{Cm}$ and the movement characteristics of the spermatozoa indicated that in some cases the linearity of the regression would be improved by transformation of the data to produce the square $(\%$ yawing) or the $\log (\mathrm{Cm}, \%$ minimal displacement, progressive mean velocity) of the observed value. Multiple regression of $\log \mathrm{Cm}$ on the movement characteristics of the spermatozoa, incorporating these transformed variables, gave a multiple $R$ value of 0.832 , on the basis of the factors indicated in Table 3 .

Table 3. Multiple regression of the log concentration of spermatozoa penetrating cervical mucus $(\mathrm{Cm})$ on the transformed movement characteristics of the spermatozoa in human semen

\begin{tabular}{lccc}
\hline \multicolumn{1}{c}{ Variable } & Multiple $R$ & $R^{2}$ change & $\begin{array}{c}\text { B } \\
\text { (non-standardized) }\end{array}$ \\
\hline Motility (\%) & 0.433 & 0.188 & $0 \cdot 103$ \\
Square of minimal displacement $(\%)$ & 0.537 & 0.099 & -0.205 \\
Progressive frequency of rotation $(/ \mathrm{sec})$ & 0.649 & $0 \cdot 133$ & -0.351 \\
Log progressive mean velocity $(\mu \mathrm{m} / \mathrm{sec})$ & 0.796 & 0.212 & 8.81 \\
Mean amplitude of displacement $(\mu \mathrm{m})$ & 0.832 & 0.059 & -0.156 \\
Constant & & & -3.669 \\
\hline
\end{tabular}

The use of $\mathrm{Cm}$ values to describe the success of sperm-cervical mucus interaction has the disadvantage that this figure does not account for differences between semen samples in the numbers of motile spermatozoa available to interact with the cervical mucus interface. Hence a 
sample possessing a high concentration of motile spermatozoa may achieve a high $\mathrm{Cm}$ score even though only a small proportion of the spermatozoa present are exhibiting the required movement characteristics. To eliminate this variable from the analysis a multiple regression was performed which used PSC $\times$ Vs as the dependent variable. Since all other components of the PSC formula are constant this dependent variable is equivalent to $\mathrm{Cm} / \mathrm{Cs}$, i.e. the concentration of spermatozoa in the cervical mucus expressed as a proportion of the concentration of motile spermatozoa in semen. Scattergram analysis indicated that the $\log$ of this dependent variable (log PSC $\times$ Vs) gave the best approximation to a linear relationship with the independent variables including the transformations used in the previous regression. This form of regression analysis revealed an extremely high correlation between sperm movement and mucus penetration yielding a multiple $R$ value of 0.874 on the basis of 5 variables of which the proportion of spermatozoa failing to exhibit significant lateral displacement was the first incorporated and most significant $\left(R^{2}=0.530\right)$ variable, exhibiting a simple correlation coefficient with the dependent variable of -0.728 $(P<0.001)$ (Table 4). Therefore, when differences between samples in the numbers of motile spermatozoa present in semen are considered, $76 \%$ of the variation in cervical mucus penetration can be accounted for by the existence of linear correlations with the variables listed in Table 4 .

Table 4. Multiple regression of $\log$ PSC $\times$ Vs against the movement characteristics of human spermatozoa

\begin{tabular}{lcccc}
\hline \multicolumn{1}{c}{ Variable } & Multiple $R$ & $R^{2}$ change & $\begin{array}{c}\text { B } \\
\text { (non-standardized) }\end{array}$ & Simple $r$ \\
\hline Square of minimal displacement $(\%)$ & 0.729 & 0.530 & 0.138 & $-0.728^{* * *}$ \\
Progressive, lateral displacement $<10 \mu \mathrm{m}(\%)$ & 0.760 & 0.047 & -0.783 & $-0.622^{* *}$ \\
Log rolling (\%) & 0.779 & 0.030 & 1.317 & $0.587^{*}$ \\
Yawing (\%) & 0.836 & 0.091 & 0.301 & 0.305 \\
Square of progressive yawing (\%) & 0.874 & 0.065 & -0.328 & $0 \cdot 135$ \\
Constant & & & $1 \cdot 165$ & \\
\hline
\end{tabular}

$* P<0.02,{ }^{* *} P<0.01,{ }^{* * *} P<0.001$

\section{Discussion}

Male fertility is conventionally assessed on the basis of a semen profile incorporating descriptive criteria, reflecting the quality of the ejaculate in terms of its overall appearance, sperm number, morphology and motility. Such descriptive criteria are adequate to identify the most severe lesions in male reproductive function, as in oligo- or azoospermia, but when the defects are more subtle, as in idiopathic infertility, prospective studies have shown that the conventional semen profile is incapable of discriminating between fertile and infertile men (Aitken et al., 1984). In contrast, techniques which focus on the functional properties of the spermatozoa, rather than their number or appearance, have been shown to be of significant prognostic value in ascertaining the fertilizing potential of a patient in vivo. Two in-vitro techniques in particular, which measure the ability of human spermatozoa to penetrate zona-free hamster oocytes (Rogers et al., 1979; Cohen, Weber, van der Vijver \& Zeilmaker, 1982; Aitken et al., 1984) and cervical mucus (Ulstein, 1973), do generate information of prognostic value in vivo.

The zona-free hamster oocyte penetration test can discriminate between fertile donors and patients exhibiting idiopathic oligozoospermia, varicocoele and unexplained infertility (Rogers $e t$ al., 1979; Aitken, 1983). The outcome of this test has also been shown to correlate with the duration of infertility in vivo as well as the incidence of pregnancy in prospective studies (Cohen et al., 1982; Aitken et al., 1984). The test is considered to be of greatest value in detecting subnormal sperm function in patients exhibiting normal semen profiles (Rogers et al., 1979), examples of which were 
present in the donor population used in this study. Since correlations have been observed between the post-capacitation movement characteristics of human spermatozoa and their performance in the hamster oocyte assay (Aitken et al., 1982a), it was of interest to determine whether the type of patient for which the penetration test is optimal (exhibiting defective sperm function in the face of a normal semen profile) could be identified on the basis of a multiple regression equation incorporating the post-capacitation movement characteristics of the spermatozoa. If such a relationship existed and, furthermore, was found to apply to the motility of human spermatozoa in semen, a simple alternative to inter-species in-vitro fertilization might have been revealed, which could have been more easily adopted as a routine diagnostic procedure than the latter.

When these possibilities were examined, it was found that multiple regression equations incorporating the post-capacitation movement characteristics of the spermatozoa were not, in fact, successful in identifying those patients exhibiting subnormal fertilizing potential in the hamster oocyte assay. Furthermore, the limited degree of correlation that did exist in this respect did not extend to the movement characteristics of the spermatozoa in semen. Therefore, while the relationship between sperm motility and fertilizing potential may be sufficiently close to be of predictive value when the semen profile is abnormal, as in oligo-asthenozoospermia (Aitken et al., $1982 \mathrm{~b}$ ), no consistent, clinically useful, relationship appears to obtain in normospermic males. This conclusion has also been emphasized in a study of patients exhibiting unexplained infertility, many of whom possess defects in the penetrating capacity of their spermatozoa without any accompanying change in the characteristics of sperm movement (Aitken et al., 1983b). In such cases specific defects in the ability of the spermatozoa to acrosome react or generate a fusogenic equatorial segment must be responsible for the impaired capacity for oocyte penetration despite a normal pattern of motility.

In contrast to the above findings, the specific motility patterns of human spermatozoa in semen exhibited a powerful correlation with their potential for cervical mucus penetration. In previous studies we have emphasized the importance of semen quality, rather than the properties of the cervical mucus in determining the success of sperm-cervical mucus interaction in infertile patients (Schats et al., 1984). While sperm motility was recognized as an important aspect of semen quality in this respect (Schats et al., 1984), the specific attributes of sperm movement that are decisive in achieving cervical mucus penetration have been identified in the present study. In this analysis any variation due to mucus quality was eliminated by using a cryostored pool of bovine cervical mucus, since this material has been shown to reflect accurately the interaction of human spermatozoa and human mucus (Bergman, Amit, David, Homonnai \& Paz, 1981; Mangione, Medley \& Menge, 1981), exhibiting correlation coefficients of 0.982 and 0.931 in independent studies (Bergman et al., 1981 ; R. J. Aitken, unpublished observations). In terms of the measurement of cervical mucus penetration, multiple regression equations incorporating the PSC scores as the dependent variable gave a poorer correlation with sperm movement than did $\mathrm{Cm}$ or $\mathrm{Cm} / \mathrm{Cs}$. This may be because the behaviour of spermatozoa cannot, as the PSC calculations assume, be accurately described in terms of the kinetic theory of gases (Ojakian \& Katz, 1973; Schats et al., 1984). The fact that one of the samples analysed in this study gave an impossible PSC score of $102 \%$ also points to inadequacies in the theoretical basis for such calculations.

When the $\mathrm{Cm}$ values were used to describe the efficiency of sperm-cervical mucus interaction, about $70 \%$ of the variation observed could be explained by differences between samples in the specific motility patterns exhibited by the spermatozoa (Table 3). The most important factors in this analysis were the linear velocity of progression (incorporated into the regression equation as $\log$ progressive mean velocity and progressive frequency of rotation), the concentration of motile cells (incorporated as $\%$ motility) and the amplitude of lateral sperm head displacement (incorporated as the square of the $\%$ of spermatozoa exhibiting minimal displacement and the mean amplitude of displacement). When differences between samples in the concentration of motile spermatozoa available to interact with the cervical mucus interface were accounted for (Table 4 ), $76 \%$ of the remaining variation of the cervical mucus penetration could be explained by differences in the 
patterns of sperm motility. Under these conditions the amplitude of lateral sperm head displacement, incorporated into the regression equation as the square of the \% of spermatozoa exhibiting minimal displacement $\left(R^{2}=0.530 ; r=0.728\right)$, again appeared to be the dominant factor determining the success of sperm-cervical mucus interaction. The relationship between cervical mucus penetration and the amplitude of lateral sperm head displacement was further emphasized in the simple and multiple regression equations utilizing the PSC scores as the dependent variable.

Adequate lateral displacement of the sperm head may be required for the spermatozoon to insinuate itself into the channels between the mucin chains. The amplitude of sperm head displacement may also indirectly reflect the efficiency of the flagellar beat pattern which, in turn, may be responsible for the failure to achieve adequate mucus penetration. The amplitude of lateral sperm head displacement is positively correlated with the size of the flagellar beating envelope (David et al., 1981). As spermatozoa become momentarily immobilized at the cervical mucus interface, large amplitude flagellar undulations would be required to generate the maximum thrust. It is possible that samples exhibiting a small amplitude of lateral sperm head displacement may also possess a small-amplitude flagellar beat pattern which is incapable of generating the thrust required to penetrate the mucus barrier. Whatever the reasons for the correlation between the amplitude of lateral sperm head displacement and cervical mucus penetration, its existence has important implications for both contraceptive development and the study of idiopathic infertility.

\section{References}

Aitken, R.J. (1983) Attributes and applications of the zona-free hamster egg penetration test. In In Vitro Fertilization and Embryo Transfer, pp. 167-193. Eds P. G. Crosignani \& B. G. Rubin. Academic Press, London.

Aitken, R.J., Best, F.S.M., Richardson, D.W., Djahanbakhch, O. \& Lees, M.M. (1982a) The correlates of fertilizing capacity in normal fertile men. Fert. Steril. 38, 68-76.

Aitken, R.J., Best, F.S.M., Richardson, D.W., Djahanbakhch, O., Mortimer, D., Templeton, A.A. \& Lees, M.M. (1982b) An analysis of sperm function in cases of unexplained infertility: conventional criteria, movement characteristics and fertilizing capacity. Fert. Steril. 38, 212-221.

Aitken, R.J., Best, F.S.M., Richardson, D.W., Djahanbakhch, O., Templeton, A. \& Lees, M.M. (1982c) An analysis of semen quality and sperm function in cases of oligozoospermia. Fert. Steril. 38, 705-711.

Aitken, R.J., Wang, Y.-F., Lui, J., Best, F. \& Richardson, D.W. (1983a) The influence of medium composition, osmolarity and albumin content on the acrosome reaction and fertilizing capacity of human spermatozoa: development of an improved zona-free hamster egg penetration test. Int. J. Androl. 6, 180-193.

Aitken, R.J., Warner, P., Best, F.S.M., Templeton, A.A., Djahanbakhch, O., Mortimer, D. \& Lees, M.M. (1983b) The predictability of subnormal penetrating capacity of sperm in cases of unexplained infertility. Int. J. Androl. 6, 212-220.

Aitken, R.J., Best, F.S.M., Warner, P. \& Templeton, A. (1984) A prospective study of the relationship between semen quality and fertility in cases of unexplained infertility. J. Androl. 5, 297-303.

Atherton, R.W., Radany, E.W. \& Polakoski, K.L. (1977) Quantitation of human sperm motility. In Techniques of Human Andrology, pp. 173-188. Ed. E. S. E. Hafez. North Holland, Amsterdam.

Bedford, J.M. (1983) Significance of the need for sperm capacitation before fertilization in eutherian mammals. Biol. Reprod. 28, 108-120.

Bergman, A., Amit, A., David, M.P., Homonnai, Z. T. \& Paz, G.F. (1981) Penetration of human ejaculated spermatozoa into human and bovine cervical mucus. 1. Correlation between penetration values. Fert. Steril. 36, 363-367.

Biggers, J.D., Whitten, W.K. \& Whittingham, D.G. (1971) The culture of mouse embryos in vitro. In Methods in Mammalian Embryology pp. 86-116. Ed. J. C. Daniel, Jr. Freeman, San Francisco.

Cohen, J., Weber, R.F.A., van der Vijver, J.C.M. \& Zeilmaker, G.H. (1982) In vitro fertilizing capacity of human spermatozoa with the use of zona-free hamster ova: interassay variation and prognostic value. Fert. Steril. 37, 565-572.

David, G., Serres, C. \& Jouannet, P. (1981) Kinematics of human spermatozoa. Gamete Res. 4, 83-95.

David, M.P., Amit, A., Bergman, A., Yedwab, G., Paz, G.F. \& Homonnai, Z.T. (1979) Sperm penetration in vitro: correlations between parameters of sperm quality and the penetration capacity. Fert. Steril. 32, 676-680.

Katz, D.F. \& Overstreet, J.W. (1981) Sperm motility assessment by videomicrography. Fert. Steril. 35, 188193.

Katz, D.F., Overstreet, J.W. \& Hanson, F.W. (1980) A new quantitative test for sperm penetration into cervical mucus. Fert. Steril. 33, 179-186.

Mangione, C.M., Medley, N.E. \& Menge, A.C. (1981) Studies on the use of estrous bovine cervical mucus in the human sperm-cervical mucus penetration technique. Int. J. Fertil. 26, 20-24. 
Mortimer, D. \& Templeton, A.A. (1982) Sperm transport in the human female reproductive tract in relation to semen analysis characteristics and time of ovulation. J. Reprod. Fert. 64, 401-408.

Nie, N.H., Hull, C.H., Jenkins, J.G., Steinbrenner, K. \& Bent, D.H. (1975) Statistical Package for the Social Sciences. McGraw Hill, New York.

Ojakian, G.K. \& Katz, D.T. (1973) A simple technique for measurement of swimming speed of chlamydomonas. Expl Cell. Res. 81, 487-491.

Overstreet, J.W., Katz, D.F., Hanson, F.W. \& Fonseca, J.R. (1979) A simple inexpensive method for objective assessment of human sperm movement characteristics. Fert. Steril. 31, 162-172.

Rogers, B.J., Van Campen, H., Ueno, M., Lambert, H., Bronson, R. \& Hale, R. (1979) Analysis of human spermatozoal fertilizing ability using zona-free ova. Fert. Steril. 32, 664-670.

Schats, R., Aitken, R.J., Templeton, A.A. \& Djahanbakhch, O. (1984) The role of cervical mucus-semen interaction in infertility of unknown aetiology. Br. $J$. Obstet. Gynaec. 91, 371-376.

Serres, C., Feneux, D., Jouannet, P. \& David, G. (1984) Influence of the flagellar wave development and propagation on the human sperm movement in seminal plasma. Gamete Res. 9, 183-195.

Ulstein, M. (1973) Fertility of donors at heterologous insemination. Acta obstet. gynaec. scand. 52, 97-101.

World Health Organization (1980) Laboratory Manual for the Examination of Human Semen and Semen-Cervical Mucus Interaction. Eds M. A. Belsey, R. Eliasson, A. J. Gallegos, K. S. Moghissi, C. A. Paulsen \& M. R. N. Prasad. Press Concern, Singapore.

Yanagimachi, R. (1970) The movement of golden hamster spermatozoa before and after capacitation. J. Reprod. Fert. 23, 193-196.

Yanagimachi, R., Yanagimachi, H. \& Rogers, B.J. (1976) The use of zona-free animal ova as a test system for the assessment of fertilizing capacity of human spermatozoa. Biol. Reprod. 15, 471-476.

Received 22 June 1984 